\section{Impression cytology with scanning electron microscopy: a new method in the study of conjunctival microvilli}

${ }^{1}$ Eye Department, University of Naples, Federico II, Naples, Italy

${ }^{2}$ Interdepartmental Electron Microscope Centre, University of Naples, Federico II, Naples, Italy

Correspondence: A Del Prete,

Istituto di Scienze Oftalmologiche,

Università di Napoli Federico

II, via Pansini 5,

Napoli 80131, Italy

Tel: + 390814138 03;

Fax: + 390812512994 .

E-mail: andelp@

tin.it

Received: 2 August 2006 Accepted in revised form: 26 April 2007

Published online: 29 June 2007

Competing Interests Statement: We have no competing interests to declare.

\begin{abstract}
Purpose Recent studies used impression cytology with scanning electron microscopy (SEM) to study the conjunctival surface of bovine eyes and normal human eyes. The purpose of this study was to evaluate the use impression cytology and SEM (ICSEM) in patients affected by tear film abnormalities. Methods Forty-five patients were divided into three groups according to mild, moderate or severe subjective sensation of dry eye. Fifteen asymptomatic subjects served as control group. In all patients the tear film was evaluated with break-up time (BUT), Schirmer's, and Ferning test, whereas conjunctival epithelium was evaluated with impression cytology and optic microscopy (ICOM), and ICSEM. The Spearman rank correlation test was used to compare the outcome of these examinations with the subjective sensation of dry eye in each group, and to identify correlations among the five tests.

Results ICSEM findings highly correlated with subjective dry eye sensation (Spearman correlation coefficient, 796; $P<0.01$ ). ICSEM revealed incipient epithelial damage (reduction or absence of microvilli) before the appearance of alterations of nucleus and cytoplasm of epithelial cells revealed by optic microscopy. The number of microvilli was correlated with the degree of tear film abnormalities and subjective sensation of dry eye (Spearman correlation coefficient, 796; $P<0.01$ ).

Conclusion ICSEM was very effective in detecting the reduction in the number of microvilli. Therefore, it could represent an effective method to detect alterations in the conjunctival epithelium resulting from tear
\end{abstract}

GL Cennamo'1, A Del Prete'1, R Forte', G Cafiero², $S$ Del Prete ${ }^{2}$ and $D$ Marasco ${ }^{2}$

film damage even before the epithelial damage occurs and is detected by optic microscopy. Eye (2008) 22, 138-143; doi:10.1038/sj.eye.6702873; published online 29 June 2007

Keywords: dry eye; conjunctival epithelium; impression cytology; microvilli; scanning electron microscopy

\section{Introduction}

Tear film abnormalities are frequently encountered in clinical practice. They can be due to alterations of the mucous, aqueous or lipid component of the tear film or to changes in the corneal-conjunctival epithelium. ${ }^{1,2}$ Various techniques are used to study tear film changes, namely break-up time (BUT), and Schirmer's and Ferning tests, and to evaluate the conjunctival epithelial cells, namely impression cytology with light microscopy (or optical microscopy, ICOM), and scanning electron microscopy (SEM) of conjunctival biopsy samples. Electron microscopy has been used in tear deficiency to evaluate ultrastructural changes of conjunctival epithelial cells. SEM has been applied to conjunctival biopsies of dry eye patients and has revealed the fine structure of cellular and nuclear changes (nuclear segmentation, abnormal chromatin distribution, reduction or loss of goblet-cells, signs of squamous metaplasia). ${ }^{3,4}$ However, its bioptic results are not reproducible. Impression cytology is widely used as a noninvasive alternative to full-thickness biopsy to obtain epithelial cells from the ocular surface. Recent studies used impression cytology with SEM to study the conjunctival surface of bovine eyes ${ }^{5}$ and normal human eyes. ${ }^{6}$ Thus far, there have 
been no SEM studies of conjunctival impression cytology samples (ICSEM) in patients affected by tear film abnormalities.

\section{Materials and methods}

Exclusion criteria for enrolment of patients were use of contact lens, infection, trauma, pregnancy, lactation, ocular surgery, or any other surgery within the previous 6 months, or severe blepharitis with meibomian gland dysfunction, blinking abnormality (eg Parkinson's disease, facial nerve palsy), severe pterygium, diabetes or uncontrolled systemic disease. Forty-five patients (23 men and 22 women; mean age 34 years; 90 eyes) affected by tear film abnormalities were enrolled in the study and were divided in three groups according to subjective dry eye sensation. The criteria to define dry eye sensation were itching, burning, irritation, and watering. Fifteen patients had mild dry eye sensation (seven women and eight men; mean age 31 years), 15 had moderate dry eye sensation (eight women and seven men; mean age 33 years), and 15 had severe dry eye sensation (seven women and eight men, mean age 38 years). Fifteen volunteers (eight men and seven women; mean age 29 years) without any sensation of dry eye were used as controls. No patient had disorders of the eye apart from tear film abnormalities. The ophthalmic examination consisted of a slit lamp examination, and determination of tear film BUT. Schirmer's test and Ferning test, impression cytology associated with optic microscopy and impression cytology associated with SEM were also performed. The results of each test have been graded from 1 to 4 according to the degree of severity, with one indicating absence of alterations. The study was in compliance with the tenets of the Declaration of Helsinki, patient participation was voluntary and informed consent to undergo the procedures was obtained from each subject.

\section{Tear function examinations}

No subject used topical artificial tear drops within $6 \mathrm{~h}$ before the examinations. Two microliters of a preservative-free combination of $1 \%$ rose bengal and $1 \%$ fluorescein dye was instilled in the conjunctival sac with a micropipette. The tear film BUT measurement was performed after instillation. The subjects were instructed to blink several times for a few seconds, to ensure adequate mixing of fluorescein. The interval between the last complete blink and the appearance of the first corneal black spot in the stained tear film was measured three times, and the mean value of the measurements was calculated. A BUT of less than $10 \mathrm{~s}$ was considered abnormal. ${ }^{7}$ The standard Schirmer's test- 1 without topical anesthesia ( $0.4 \%$ oxybuprocaine chloride) was performed. The standardized strips of filter paper (Alcon Inc., Fort Worth, TX, USA) were placed in the lateral canthus away from the cornea and left in place for $5 \mathrm{~min}$ with the eyes closed. Readings were reported in millimetres of wet strip after $5 \mathrm{~min}$. A reading of less than $5 \mathrm{~mm}$ was considered to show dry eye. Ferning test was performed and scored. ${ }^{8}$ Score $>$ II/III was regarded as pathological.

\section{Impression cytology, light microscopy and SEM}

Three days after tear function examinations, an impression cytologic specimen for light microscopy was obtained. After 1 week, another specimen for SEM was obtained in the same conjunctival area. Conjunctival epithelium specimens were collected, without anaesthetic from patients and control subjects by compressing a fragment of cellulose acetate on the bulbar upper-temporal conjunctival surface for 3 or $4 s ; ;^{9}$ specimens were transferred to a glass slide by compressing the cellulose acetate fragment on the glass slide for $30 \mathrm{~s}$. To perform light microscopy, they were dehydrated in a crescent series of alcohol up to $95 \%$ and stained with alcian blue/fucsina, analysed and photographed under a Axioskop Zeiss microscope.

For SEM, the conjunctival epithelial samples were fixed in $3 \%$ glutaraldehyde in a $0.065 \mathrm{M}$ ( $\mathrm{pH} 7.4$ ) phosphate buffer for $2 \mathrm{~h}$ at room temperature. Slides were washed three times in $0.065 \mathrm{M}$ phosphate buffer (for $30 \mathrm{~min}$ ), then placed in $1 \% \mathrm{OsO} 4$ in $0.064 \mathrm{M}$ (pH 7.4) phosphate buffer for $30 \mathrm{~min}$. Samples were dehydrated through a graded series of ethanol, and then criticalpoint-dried in a $\mathrm{CO}_{2}$ liquid Bemar SPC 1500 apparatus (Bomar Co, Tacome, WA, USA). Specimens were mounted on aluminium stubs with silver-conducting paint, sputtered with a thin $(20 \mathrm{~nm})$ film of gold and observed with a Cambridge Mark 250 SEM. The microvilli on each sample were first identified at a magnification of $\times 750$ in a $1500 \mu \mathrm{m}^{2}$ field. Then, at a magnification of $\times 7500$, we counted the number of microvilli in each $230 \mu \mathrm{m}^{2}$ area of the field selected.

\section{Statistics}

To quantify the correlations among the five tests and the differences between the three groups of patients and the control group, we analysed the test results (ranked from 1 to 4 ) using Spearman rank correlation. All $P$-values refer to a two-sided test and a $P$-value $\leq 0.01$ was considered statistically significant. The calculations were performed using the SPSS package (SPSS for Windows 12.0: SPSS Inc., Chicago, IL, USA). 


\section{Results}

Most $(63 \%)$ of the 15 control subjects had a score of 1 on each test, and $37 \%$ had a score indicative of mild alteration. An absence of goblet cells was found at ICOM examination in eight $(26.6 \%)$ of the 30 control eyes.

In the 30 eyes presenting mild subjective symptoms, the BUT test was negative in 10 eyes (33\%), positive in nine $(30 \%)$ eyes and highly positive in 11 eyes (36.6\%). The Schirmer's test results were normal in 11 eyes (36.6\%), mildly altered (between 5 and $10 \mathrm{~mm}$ ) in 18 eyes $(60 \%)$, and severely altered in one eye (between 0 and $5 \mathrm{~mm}$ ) (3.3\%). There was full ferning in one eye (3.3\%), partial ferning with mild abnormalities in 14 eyes (46.6\%), and partial ferning with small ferns in 15 eyes (50\%). ICOM showed an absence of goblet cells in all patients with mild tear film abnormalities. There were some apparently normal epithelial cells in five eyes $(16.6 \%)$, whereas the nucleus and cytoplasm of epithelial cells were reduced in size in 16 eyes (53.3\%). Deformed cells with snake-like nuclei were identified in nine eyes (30\%). SEM revealed a slightly reduced number of microvilli in 11 eyes (36.6\%), and heavy reduction of microvilli in the remaining 19 eyes (63.3\%).

In the 30 eyes with moderate dry eye symptoms, the BUT test was negative in 14 eyes $(46.6 \%)$, slightly positive in three eyes (10\%), and highly positive in 13 eyes $(43.3 \%)$. The Schirmer's test was normal in three eyes $(10 \%)$, slightly altered in 11 eyes $(36.6 \%)$ and severely altered in 16 eyes $(53.3 \%)$. There were small ferns in 17 eyes $(56.6 \%)$ and partial ferning in 13 eyes $(43.3 \%)$. ICOM showed an absence of goblet cells in all patients of this group. In addition, epithelial cells with a small nucleus and cytoplasm were found in 13 eyes $(43.3 \%)$, and deformed cells with snake-like nuclei were detected in the remaining 17 eyes (56.6\%). ICSEM revealed a mild reduction in the number of microvilli in 11 eyes $(36.6 \%)$, and a heavy reduction of microvilli in 19 eyes $(63.3 \%)$.

In the 30 eyes presenting severe dry eye symptoms, the BUT test was slightly positive in seven eyes $(23.3 \%)$ and highly positive in $23(76.6 \%)$ eyes. The Schirmer's test results were mildly altered in nine eyes $(30 \%)$ and severely altered in $21(70 \%)$ eyes. There were small ferns in 15 eyes (50\%) and no ferning in the remaining 15 eyes (50\%). ICOM showed an absence of goblet cells in all patients of this group. Epithelial cells with a small nucleus and cytoplasm occurred in five eyes (16.6\%), deformed cells with a snake-like nuclei in 12 eyes (40\%), an absence of nucleus in 13 eyes (43.3\%). ICSEM showed a heavy reduction of microvilli in 11 eyes $(36.6 \%)$ and damaged epithelial cells in 19 eyes (63.3\%).

To detect the presence of a monotone association between the subjective dry eye sensation and instrumental data, we compared the test results using Spearman rank correlation coefficient. Table 1 summarizes the strength of correlation between the tear film and conjunctival alterations detected by the five tests and the subjective dry eye symptoms. The correlations found are reported in an increasing degree of association with subjective symptoms: BUT test (Spearman's correlation coefficient, 483; $P<0.01$ ), Schirmer's test (Spearman correlation coefficient, 705; $P<0.01$ ), impression cytology evaluated with SEM (Spearman correlation coefficient, 796; $P<0.01$ ), Ferning test (Spearman correlation coefficient, 808; $P<0.01$ ), impression cytology evaluated with optic microscopy (Spearman correlation coefficient, 837; $P<0.01$ ). The number of microvilli counted with SEM was higher in the control group and progressively decreased in the three groups of patients in correlation with the severity of dry eye symptoms (Figure 1i). The Spearman rank correlation coefficient among the results of the five tests showed high correlation among Ferning test, ICOM and ICSEM, whereas Schirmer's test and BUT showed a progressively lower correlation with the other tests (Table 2).

\section{Discussion}

This is the first time that SEM has been used in association with impression cytology to evaluate tear film abnormalities. Microvilli in eyes with tear film changes have previously been studied with SEM applied to conjunctival biopsy ${ }^{10}$ (Figure 2), which is an invasive technique and is not invariably reproducible. ${ }^{4,9,11}$ On the contrary, impression cytology is non-invasive, easily reproducible and has the advantage that the sampled area can be subsequently re-examined during follow-up. The results of this study demonstrate that both ICSEM and ICOM are highly effective in identifying and staging tear film abnormalities.

In this study, ICSEM had a high sensitivity in recognizing a progressive reduction of epithelial

Table 1 Correlation between the degree of tear film and conjunctival alterations detected by the five tests and the degree of subjective dry eye symptoms

\begin{tabular}{llll}
\hline Spearman's $\rho$ & $\begin{array}{l}\text { Symptoms } \\
\text { Fluorescein } \\
\text { Symptoms }\end{array}$ & Correlation coefficient & 0.483 \\
& $\begin{array}{l}\text { Schirmer } \\
\text { Symptoms }\end{array}$ & Correlation coefficient & 0.705 \\
& $\begin{array}{l}\text { Ferning } \\
\text { Symptoms }\end{array}$ & Coefficient & 0.808 \\
& $\begin{array}{l}\text { ICOM } \\
\text { Symptoms } \\
\text { ICSEM }\end{array}$ & Correlation coefficient & 0.837 \\
& & 0.796 \\
\hline
\end{tabular}




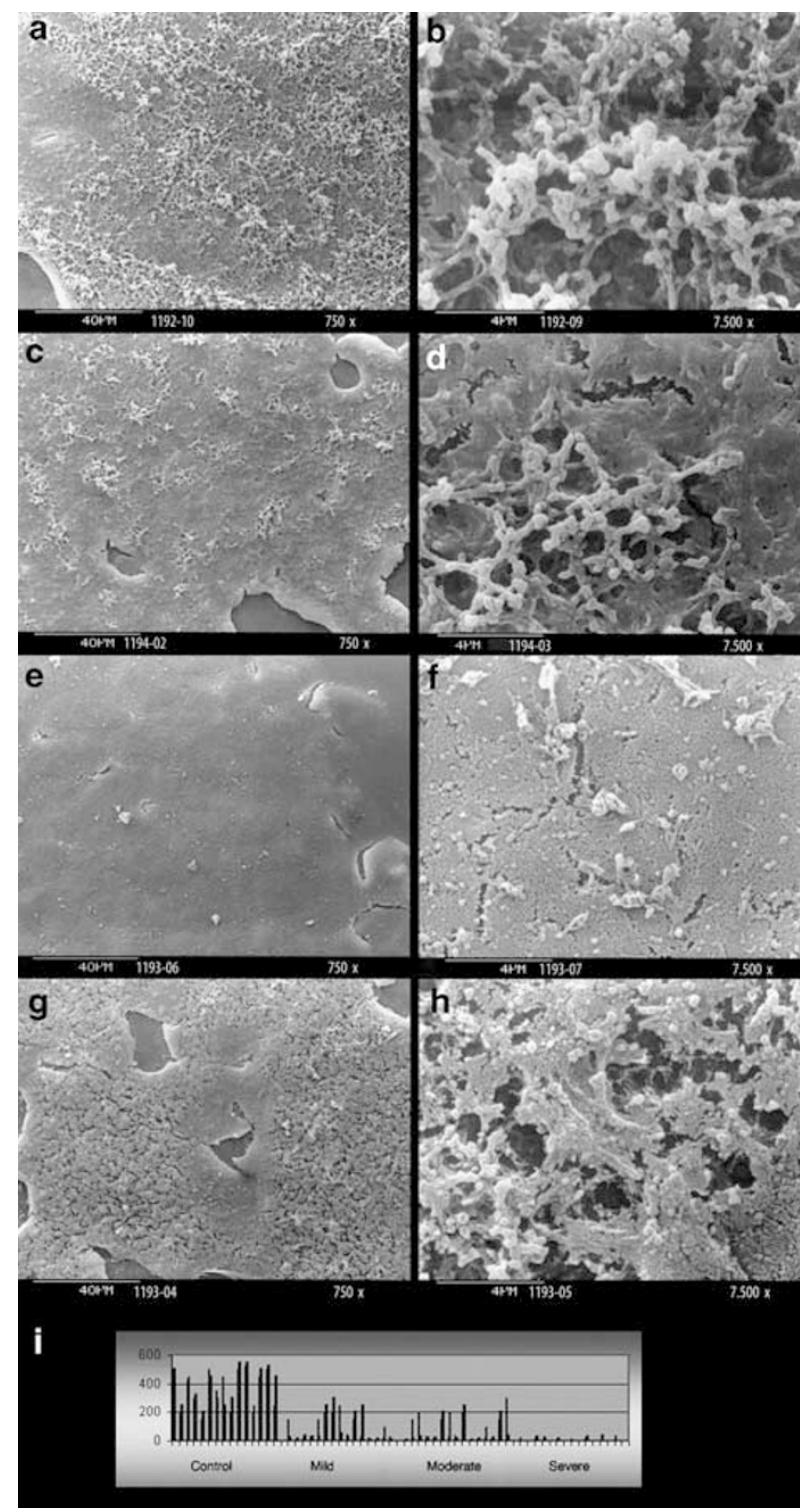

Figure 1 Scanning electron micrographs showing conjunctival epithelium evaluated with SEM applied to impression cytology. Conjunctival epithelium of a control subject. (a) $\times 750$; (b) $\times 7500$. Conjunctival epithelium of a patient affected by mild tear film damage. (c) $\times 750$; (d) $\times 7500$. Note the decrease in microvilli density compared with the control subject. Conjunctival epithelium of a patient affected by moderate tear film damage (e) $\times 750$; (f) $\times 7500$. The microvilli have almost completely disappeared. Conjunctival epithelium of a patient affected by severe tear film damage $(\mathrm{g}) \times 750 ;(\mathrm{h}) \times 7500$. Note the breakdown of epithelial cells. (i) Number of microvilli, counted in a randomly selected standard area of a magnified SEM $(\times 7500)$ micrograph, in controls and in the four groups of subjects studied.

microvilli in relation with the increasing severity of dry eye syndrome.

The mechanism underlying tear film abnormalities has yet to be clarified although an alteration of epithelial microvilli has been implicated in this process. The apical membrane of the superficial epithelial cells is covered with microvilli, which are themselves covered by glycocalyx (ocular surface glycocalyx), that represent the deepest layer of the tear film. ${ }^{1}$ Transmembrane mucins anchored at the tips of the microvilli constitute the glycocalyx. Both transmembrane and soluble mucins constitute the mucous layer. ${ }^{12,13}$ Microvilli, which form microplicae ${ }^{8,14,15}$ are present in many epithelial cells, mostly in those specialized in absorption, where they can increase the absorbing surface by as much as 30 times. The function of the microvilli and microplicae of the conjunctival epithelium is closely related to tear film stability, which in turn depends on the chemical-physical interaction of the three tear film layers. The deepest layer, namely, the mucous layer, acts as surfactant and reduces superficial tension so that the thin lipid layer is evenly distributed over the aqueous layer. ${ }^{2}$ The tear film binds to the microvilli of the epithelium surface, thereby ensuring its wettability. ${ }^{16,17}$

Tear film instability corresponds to changes in the amount and structure of microvilli. ${ }^{18,19}$ Microvilli, microplicae, and glycocalyx of the conjunctival epithelium provide support for the tear film ${ }^{20}$ and prevent defluxion by gravity of the tear film from the cornea. However, other investigators believe that the tear film remains stable thanks to the surface tension of its components, ${ }^{21}$ a concept that implicates the exchange of nutrients among microvilli in tear film stability.

The apical pole of goblet cells forms a cavity, which, depending on the stage of the secretory cycle, is either empty or filled with a mucus plug. The preocular mucus is produced by the conjunctival goblet cells. A 'second mucus secretory system' (SMS) of the conjunctiva is represented by the mucus extruded by subsurface vesicles in the non-goblet epithelial cells, that adheres to the microvilli of the corneal and conjunctival epithelial cells thereby providing a mechanical support. ${ }^{22,23}$ According to Liotet $e t a^{24}$ in damaged epithelial cells, the production of the anchorage system of tear discharge to the cellular surface becomes deficient and the corneoconjunctival epithelium loses its wettability thereby preventing the formation of a stable tear film.

A study carried out in patients with dry eyes showed a slight change in the shape of microvilli and a decrease in the number of goblet cells. ${ }^{9,10}$ Riboflavin, zinc, vitamin $\mathrm{E}$, vitamin $\mathrm{A}$, and magnesium deficiency was found to cause alterations of microvilli and goblet cells of the corneo-conjunctival epithelium in rats. ${ }^{25-27}$ This suggests that the exchange of nutrients between the tear film and the conjunctival epithelium is altered in this condition. However, it is not known whether the alteration originates in microvilli or goblet cells. 
Table 2 Correlations among the five tests

\begin{tabular}{llllcccc}
\hline & & & \multicolumn{3}{c}{ Correlations } \\
\cline { 4 - 8 } & & & BUT & Schirmer & Ferning & ICOM & ICSEM \\
\hline Spearman's $\rho$ & BUT & Correlation Significance & 1 & 0.27 & 0.39 & 0.26 & 0.39 \\
& Schirmer & Correlation Significance & 0.27 & 1 & 0.54 & 0.62 & 0.62 \\
& Ferning & Correlation Significance & 0.39 & 0.54 & 1 & 0.72 & 0.69 \\
& ICOM & Correlation Significance & 0.26 & 0.62 & 0.72 & 1 & 0.72 \\
& ICSEM & Correlation Significance & 0.39 & 0.62 & 0.69 & 0.72 & 1 \\
\hline
\end{tabular}

Correlation is significant at the 0.01 level (two-tailed).

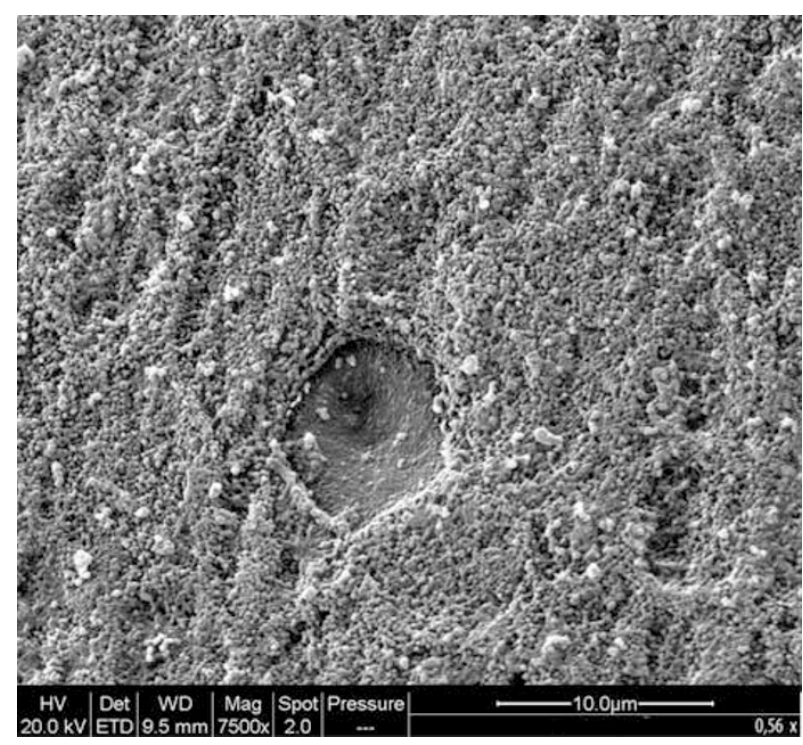

Figure 2 Electron micrograph showing normal conjunctival epithelium evaluated with SEM applied to bioptic sample, with presence of a goblet cell.

In this study, the severity of tear film abnormality seems in association with the damage to the conjunctival epithelial cells, which in turn is related to the number of microvilli. Therefore, tear film stability ultimately depends on the number of microvilli. The number of microvilli has shown to be reduced in all patients with sensation of dry eye. Furthermore, our results show that a marked decrease in the number of microvilli is followed by a reduction of conjunctival goblet cells. Both cytological abnormalities and tear film abnormalities could arise from a common, still unknown source. Alternatively, we could postulate a possible causal pathway, that is, reduction of the exchange of nutrients between epithelial cells and the tear film with a consequent decrease in absorbance, following reduction of release of intracytoplasmatic vesicle elements, which results in a reduction of the SMS and alteration of tear film stability. This would lead to a decrease in the number of microvilli because of impaired nutritional exchange, which results in further tear film instability because of lack of anchorage of the mucus component to the conjunctival epithelium. A decrease of goblet cells, which results in a reduction in mucus production, could determine further instability of the tear film.

ICSEM was found to be highly effective in classification and staging of tear film abnormalities. This technique also reveals alterations in the conjunctival epithelium resulting from tear film damage even before the epithelial damage occurs and is detected by optic microscopy. Therefore, examination of at-risk patients with ICSEM may be useful in early detection and prevention of epithelial damages.

\section{Acknowledgements}

We thank Jean Gilder for text and language editing.

\section{References}

1 Nichols B, Dawson CR, Togni B. Surface features of the conjunctiva and cornea. Invest Ophthalmol Vis Sci 1983; 24(5): 570-576.

2 Dilly PN. Contribution of the epithelium to the stability of the tear film. Trans Ophthalmol Soc UK 1985; 104(Part 4): 381-389.

3 Meller D. The fine structure of chromatin alterations in conjunctival epithelial cells in keratoconjunctivitis sicca. Cornea 1999; 18(2): 225-232.

4 Versura P, Maltarello MC, Stecher F, Caramazza R, Laschi R. Dry eye before and after therapy with hydroxypropyl methylcellulose. Ultrastructural and cytochemical study in 20 patients. Ophthalmologica 1989; 198(3): 152-162.

5 Doughty MJ. Surface features and morphology of bulbar conjunctival cells of bovine eyes obtained from a slaughterhouse: a scanning electron microscope and impression cytology study. Curr Eye Res 2002; 24(5): 341-353.

6 Kruse FE, Schmitz W, Jaeger W, Gotz ML. Scanning electron microscopy image of the cells of the conjunctival epithelium in specimens from impression cytology. Klin Monatsbl Augenheilkd 1986; 188(1): 29-32.

7 Guillon JP. Current clinical techniques to study the tear film and tear secretions. In: Korb D, Craig J, Doughty M, Guillon JP, Smith G, Tomlinson A (eds). The Tear Film. Butterworth-Heinemann: London, 2002, pp 51-83. 
8 Ravazzoni L, Ghini C, Macri A, Rolando M. Forecasting of hydrophilic contact lens tolerance by means of tear ferning test. Graefe's Arch Clin Exp Ophthalmol 1998; 236: 354-358.

9 Pfister RR, Burstein NL. The normal and abnormal human corneal epithelial surface: a scanning electron microscope study. Invest Ophthalmol Vis Sci 1977; 16(7): 614-622.

10 Versura P, Bonvicini F, Caramazza R, Laschi R. Scanning electron microscopy study of human cornea and conjunctiva in normal and various pathological conditions. Scan Electron Microsc 1985; (Pt 4): 1695-1708.

11 Rolando M, Zierhut M. The ocular surface and tear film and their dysfunction in dry eye disease. Surv Ophthalmol 2001 45(Suppl 2): S203-S210.

12 Gipson IK. Distribution of mucins at the ocular surface. Exp Eye Res 2004; 78(3): 379-388.

13 Gipson IK, Argueso P. Role of mucins in the function of the corneal and conjunctival epithelia. Int Rev Cytol 2003; 231: $1-49$.

14 Chen HB, Ou B, Yamabayashi S, Ohno S, Tsukahara S. Ultrastructural study on rat precorneal tear film by quick freezing-freeze substitution method. Nippon Ganka Gakkai Zasshi 1996; 100(6): 453-457.

15 Collin SP, Collin HB. A comparative SEM study of the vertebrate corneal epithelium. Cornea 2000; 19(2): 218-230.

16 Amemiya T. The eye and nutrition. Jpn J Ophthalmol 2000; 44(3): 320

17 Baudouin C. The pathology of dry eye. Surv Ophthalmol 2001; 45(Suppl 2): S211-S220.

18 Pfister RR, Burstein N. The effects of ophthalmic drugs, vehicles, and preservatives on corneal epithelium: a scanning electron microscope study. Invest Ophthalmol 1976; 15(4): 246-259.

19 Paterakis N, Petroutsos G, Sherrard E. Soft contact lenses and the corneal epithelium: experimental study with the scanning microscope. J Fr Ophtalmol 1987; 10(6-7): 413-417.

20 Nichols B, Dawson CR, Togni B. Surface features of the conjunctiva and cornea. Invest Ophthalmol Vis Sci 1983; 24: 570-576.

21 Holly FJ, Lemp MA. Tear physiology and dry eyes. Surv Ophthalmol 1977; 22(2): 69-87.

22 Doughty MJ. Changes in cell surface primary cilia and microvilli concurrent with measurements of fluid flow across the rabbit corneal endothelium ex vivo. Tissue Cell 1998; 30(6): 634-643.

23 Greiner JV, Henriquez AS, Weidman TA, Covington H, Allansmith MR. 'Second' mucus secretory system of the human conjunctiva. Invest Ophthal Vis Sci 1979; 18, ARVO (Suppl): 123.

24 Liotet S, Van Bijsterveld OP, Kogbe O, Laroche L. A new hypothesis on tear film stability. Ophthalmologica 1987; 195(3): 119-124.

25 Fujikawa A, Gong H, Amemiya T. Vitamin E prevents changes in the cornea and conjunctiva due to vitamin A deficiency. Graefes Arch Clin Exp Ophthalmol 2003; 241(4): 287-297. E-pub 15 March 2003.

26 Takami Y, Gong H, Amemiya T. Riboflavin deficiency induces ocular surface damage. Ophthalmic Res 2004; 36(3): 156-165. 21.

27 Gong H, Takami Y, Amemiya T, Tozu M, Ohashi Y. Ocular surface in Zn-deficient rats. Ophthalmic Res 2004; 36(3): 129-138. 